\title{
Validation of important fission product evaluations through CERES integral benchmarks
}

\author{
C.J. Dean ${ }^{\text {a }}$, P.J. Smith, and R.J. Perry
}

Serco Assurance, Winfrith Technology Centre, Dorchester DT2 8DH, UK

\begin{abstract}
Optimization of energy resources suggests increased fuel residence in reactor cores and hence improved fission product evaluations are required. For thermal reactors the fission product cross sections in the JEF2.2 and JEFF3.1 libraries plus new evaluations from WPEC23 are assessed through modelling the CERES experiment in the DIMPLE reactor. The analysis uses the lattice code WIMS10. Cross sections for 12 nuclides are assessed. The thermal cross section and low energy resonance data for ${ }^{147,152} \mathrm{Sm}$ and ${ }^{155} \mathrm{Gd}$ are accurate to within $4 \%$. Similar data for ${ }^{109} \mathrm{Ag},{ }^{143} \mathrm{Nd}$ and ${ }^{149} \mathrm{Sm}$ are within $8 \%$ while ${ }^{95} \mathrm{Mo},{ }^{99} \mathrm{Tc},{ }^{103} \mathrm{Rh},{ }^{133} \mathrm{Cs}$ and ${ }^{145} \mathrm{Nd}$ are within three benchmark standard deviations at $\sim 12 \%$. The use of the 172 XMAS group scheme is adequate for all nuclides considered except ${ }^{153} \mathrm{Eu}$.
\end{abstract}

\section{Introduction}

Optimization of energy resources suggests increased fuel residence in modern reactors. This, in turn, increases the importance of the nuclear data for fission products in the irradiated fuel. For thermal reactors the quality of the fission product cross sections in JEF2.2 [1] was assessed through benchmarking the CERES experiment [2]. This resulted in accurate evaluations being absorbed into the new JEFF-3.1 library [3] while others were either adjusted or chosen from elsewhere. In other cases new measurements were made together with further evaluation. At the same time the international approach was to develop a new fission product library from newly evaluated data for important fission products, supported by selection of others from existing evaluations. This work was achieved through sub-groups 21 and 23 of the Working Party on Evaluation Collaboration (WPEC23) [4]. This paper re-applies the CERES benchmark to JEFF3.1 and WPEC evaluations for 12 of the leading fission products.

\section{Selection of important fission products}

The fission products were selected by considering their neutron absorption impact on burn-up credit. Inventory calculations in France and the UK co-ordinated a list of fission products present in $4.32 \%$ enriched PWR fuel irradiated to $30 \mathrm{GWd}$ /te and cooled for 5 years. The fission products were listed in descending order of their absorption contribution to reactivity. It was possible to manufacture natural uranium samples doped in solutions enriched with 12 of the leading fission products. These represent $\sim 75 \%$ of fission product neutron absorption in cooled fuel for criticality interest and $\sim 50 \%$ of absorption in the reactor fuel after 2 years residence.

\footnotetext{
${ }^{a}$ Presenting author,

e-mail: christopher.dean@sercoassurance.com
}

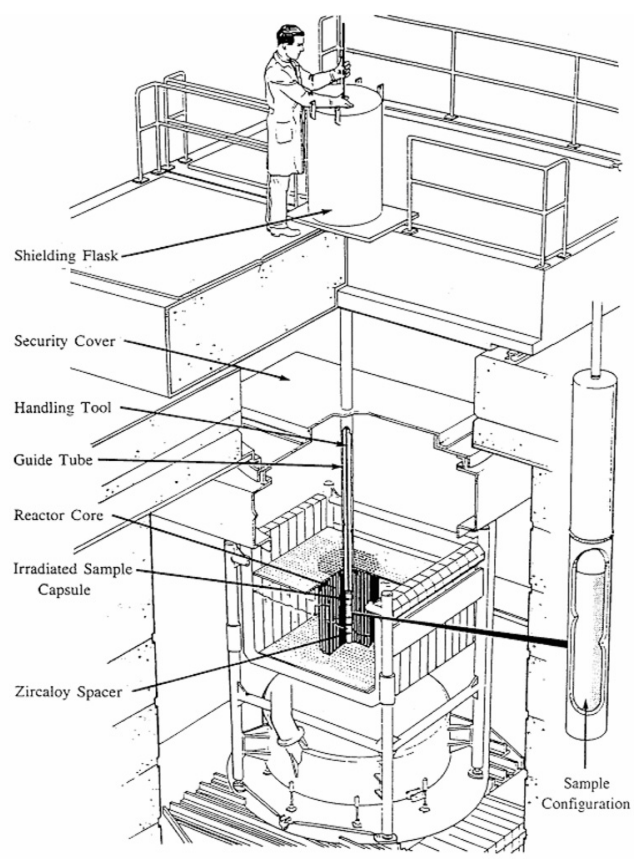

Fig. 1. Sample irradiation in DIMPLE.

\section{The CERES experiment}

The sample irradiation experiments were performed in the MINERVE reactor at Cadarache and the DIMPLE reactor (considered here) at Winfrith. Figure 1 shows the overall arrangements for the sample measurements.

As well as the samples doped with fission products a set of calibration samples, needed for the analysis, were irradiated. For each sample a set of reactivity measurements was made, where the sample was cycled between "fully out" and "fully in". At each position counts are taken from two experimental neutron detectors as a function of time to determine the asymptotic reactor period from which the core reactivity was obtained through the Inhour Equation, using calculated 


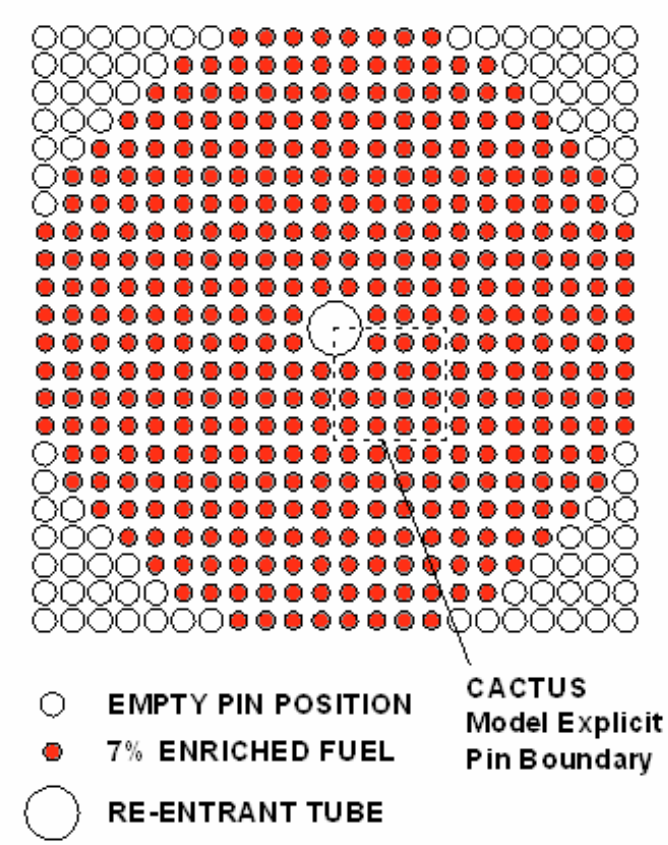

Fig. 2. CERES ASSEMBLY II: PWR spectrum.

delayed neutron parameters. The reactivity worth of the samples was deduced by subtracting the "fully in" reactivity from the mean of the preceding and succeeding "fully out" reactivities or vice versa.

The reactivity worth of the doped sample relative to an un-doped calibration sample is fairly small being of order 10$50 \mathrm{pcm}$ with an uncertainty less than $0.4 \mathrm{pcm}$. This effectively precludes the use of Monte Carlo for the analysis.

Two reactor cores were assembled to study the fission product reactivity worths. In Assembly II (fig. 2) the central re-entrant tube is surrounded by $7 \%$ enriched $\mathrm{UO}_{2}$ pins on a $1.32 \mathrm{~cm}$ square pitch yielding a spectrum close to that in PWR fuel.

In Assembly III (fig. 3) a tank of heavy water surrounds the re-entrant tube. The core is driven by $3 \%$ and $7 \%$ enriched pins to yield a thermalised (or soft) spectrum. The 7\% enriched pins are the same as those in Assembly II.

\section{Nuclear data processing}

The NJOY code [5] was used to generate 172 group cross sections for each of the 12 fission products and some others present as impurities in the dopant solutions. Above $4 \mathrm{eV}$ resonance integrals, for use in the WIMS [6] self-shielding methods, were calculated as a function of environment. Below $4 \mathrm{eV}$ the group scheme is generally adequate for WIMS to fully represent the most thermal resonances. The new fission product data replaced those on the WIMS9 Nuclear Data library [6] creating three libraries; one with JEF2.2 data, one with the fission products from JEFF3.1 and one with the fission products from WPEC23.

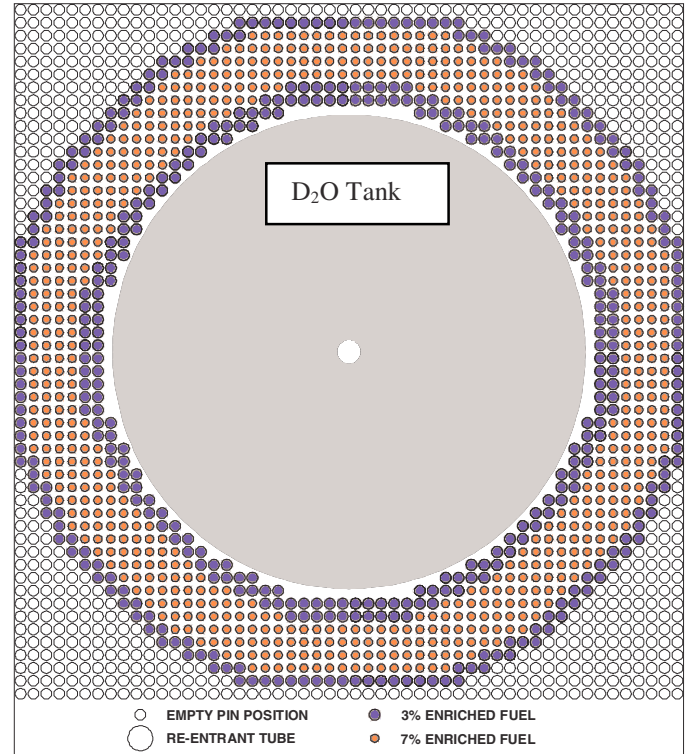

Fig. 3. CERES ASSEMBLY III: Soft spectrum.

\section{WIMS10 analysis}

The use of the ANSWERS reactor physics code WIMS to perform k-effective calculations representing CERES Assemblies II and III is well established. It has changed considerably since the time of the original CERES analyses with WIMS7. Many of the previously used WIMS modules have been updated. The WIMS10 calculation route is appropriately improved from that used in the WIMS7 analysis. In particular this analysis uses the 172 group XMAS scheme replacing the previous 69 group studies. Where appropriate the SNAP diffusion theory module, previously used to calculate the neutron importance, is replaced by CACTUS applying transport theory through the characteristics method. Resonance shielding of the scatter cross section extends the previous application to absorption and is now applied from $4 \mathrm{eV}$ to $183 \mathrm{keV}$. A fine group treatment with 100 groups per XMAS group is also available.

WIMS10 is used to calculate cross sections smeared over the sample and holder in 15 energy groups together with the neutron flux and importance. The calculation route for Assembly III is unchanged from that previously applied in WIMS7 because characteristics methods are inappropriate for studying the large heavy water region surrounding the sample. For Assembly II full advantage can be taken of the latest WIMS10 methods as follows:

The HEAD module applies Equivalence theory for less important nuclides.

PRES/CACTUS/RES modules apply sub-group resonance shielding for U-235, U-238 and the sample fission product.

PERSEUS/PIP modules calculate a 172 group condensation spectrum for use in CONDENSE.

The 15 group cross sections are used in 2D CACTUS to form the neutron flux and importance.

The CRICTIC/SMEAR combination normalises the K-eff to unity (as expected from the experiment) and produce the cross sections for exact perturbation theory. 


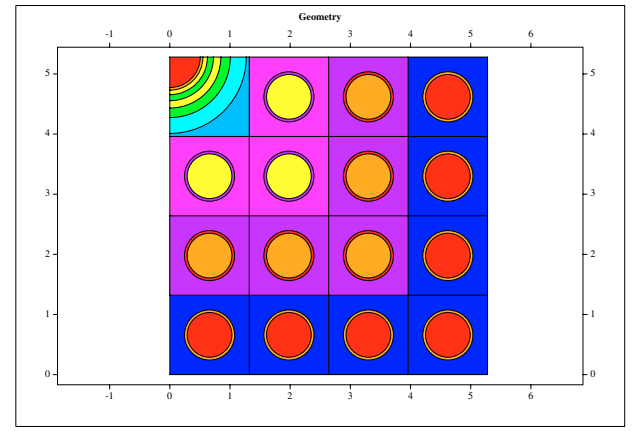

Fig. 4. WIMS10 model of Assembly II using CACTUS.

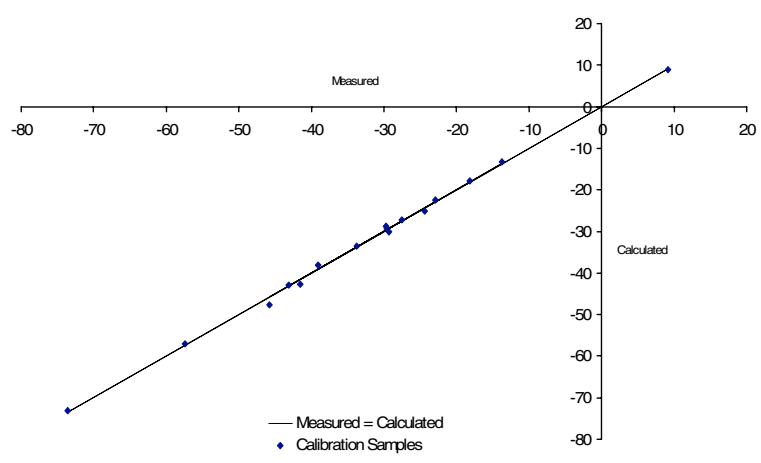

Fig. 5. Calibration for Assembly II - PWR spectrum.

The geometric model represents the sample and surrounding pins as indicated in figure 2 and expanded in figure 4.

The perturbation code for the CERES analysis yields total reactivity worths for each irradiated sample. However these are not in absolute units. A least-squares fit of calculated to measured reactivity worths both scales the calculated worths to the experimental results and achieves the expected linear fit in figure 5 for the calibration samples.

These calibration samples are variously enriched $\mathrm{UO}_{2}$ cylinders surrounded by sleeves of copper or steel loaded into zirconium sample holders. They are $10 \mathrm{~cm}$ in length. The cross sections for the components are believed to be well known relative to those of the fission products. The calibration for both assemblies is believed to be excellent. The reactivity worth of each fission product sample is obtained from the same analysis and is always relative to an un-doped reference sample. In figure 6 they are plotted against the calibration line previously obtained from the calibration for Assembly II.

Similar calibration and fission product reactivity worths are obtained for Assembly III with its soft spectrum.

\section{Results}

The experimental reactivity worths are listed in table 1 .

The change in each small reactivity worth with spectrum can be clearly seen. It can be attributed to the size and position of the dominant thermal resonances relative to $0.025 \mathrm{eV}$ where the adequacy of the thermal cross section is often the dominant effect. An assessment of the accuracy of the overall benchmark indicates $\sim 4 \%$ is appropriate. The second column of table 1

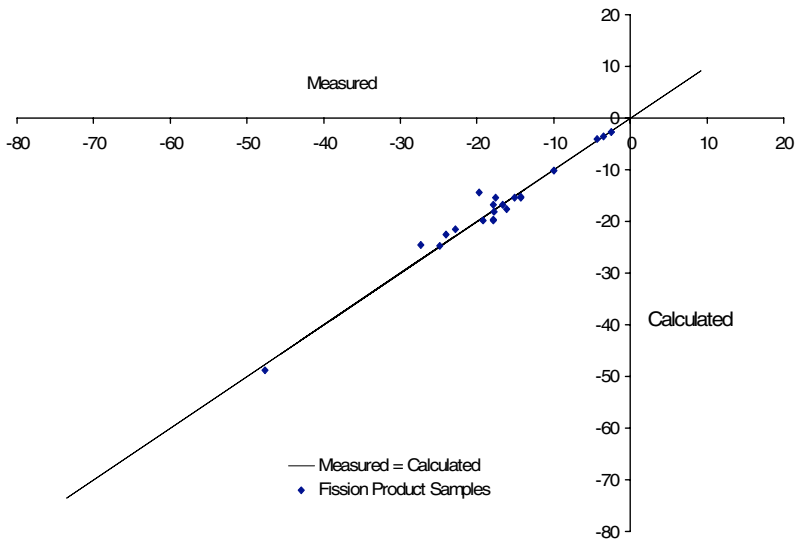

Fig. 6. Fission product worths in Assembly II: PWR spectrum.

Table 1. Measured Reactivity worth (pcm) of samples enriched in individual fission products.

\begin{tabular}{|ccll|}
\hline Fission Product & Accuracy & PWR & SOFT \\
Mo-95 & $3 \sigma$ & -9.94 & -9.15 \\
Tc-99 & $3 \sigma$ & -15.07 & -10.01 \\
Rh-103 & $3 \sigma$ & -16.11 & -9.37 \\
Ag-109 & $2 \sigma$ & -17.81 & -10.46 \\
Cs-133 & $3 \sigma$ & -17.88 & -11.62 \\
Nd-143 & $2 \sigma$ & -17.91 & -20.96 \\
Nd-145 & $3 \sigma$ & -16.62 & -14.97 \\
Sm-147 & $1 \sigma$ & -47.66 & -43.80 \\
Sm-149 & $2 \sigma$ & -24.02 & -27.07 \\
Sm-152 & $1 \sigma$ & -24.89 & -20.89 \\
Eu-153 & Method & -27.33 & -19.14 \\
Gd-155 & $1 \sigma$ & -19.27 & -29.49 \\
\hline
\end{tabular}

Table 2. Percentage discrepancy in reactivity worth for Assembly II.

\begin{tabular}{|cccc|}
\hline Fission Product & JEF2.2 & JEFF3.1 & WPEC23 \\
Mo-95 & +2 & 0 & 0 \\
Tc-99 & +3 & +8 & +10 \\
Rh-103 & +10 & +6 & +8 \\
Ag-109 & +2 & +2 & +2 \\
Cs-133 & +10 & +10 & +10 \\
Nd-143 & -6 & -3 & -6 \\
Nd-145 & 0 & +1 & +11 \\
Sm-147 & +2 & +4 & 0 \\
Sm-149 & -6 & -4 & -6 \\
Sm-152 & 0 & 0 & 0 \\
Eu-153 & -10 & -6 & -6 \\
Gd-155 & +3 & +3 & +3 \\
\hline
\end{tabular}

gives an indication of which fission products have data believed accurate within factors of this standard deviation.

The difference between experimental and predicted reactivity worth for each fission product is given in table 2 for the PWR type spectrum and in table 3 for the soft spectrum.

\section{Analysis}

The JEF2.2 results are similar to those previously reported [1]. Following previous CERES analysis, JEFF3.1 included 
Table 3. Percentage discrepancy in reactivity worth for Assembly III.

\begin{tabular}{|cccc|}
\hline Fission Product & JEF2.2 & JEFF3.1 & WPEC23 \\
Mo-95 & +11 & +9 & +9 \\
Tc-99 & -3 & +9 & +10 \\
Rh-103 & +13 & +10 & +12 \\
Ag-109 & +5 & +5 & +5 \\
Cs-133 & +12 & +11 & +11 \\
Nd-143 & -3 & -1 & -2 \\
Nd-145 & 0 & +1 & +13 \\
Sm-147 & +5 & +7 & +3 \\
Sm-149 & 0 & +2 & 0 \\
Sm-152 & -1 & 0 & -1 \\
Eu-153 & -17 & -11 & -11 \\
Gd-155 & +4 & +4 & +4 \\
\hline
\end{tabular}

Ag-109, Nd-145, Sm-147, Sm-152 and Gd-155 evaluations from JEF2.2 as indicated by minimal changes in tables 2 and 3 . The improvement for $\mathrm{Nd}-143$ can be attributed to the purposeful $4 \%$ increase in neutron width for the bound level and that for Sm-149 to the 3\% increase in the total width for the first resonance. Adoption of ENDF/B-VII data for Mo95 improves Assembly II results but table 3 suggests thermal data need attention. The choice of ENDF/B-VIr7 for Cs-133 reduces the height of the $5.9 \mathrm{eV}$ resonance but also slightly lowers its energy resulting in no improvement. Tests with WIMS fine group treatment had minimal effects indicating resonance overlap was not the cause of the discrepancy. The poor representation of the resonances at 2.5, 3.3 and $3.9 \mathrm{eV}$ in Eu-153 by the XMAS structure suggests the results may not be meaningful. The considerable effort in producing a new Rh-103 evaluation has improved results but the discrepancy still needs reducing. Similar efforts for Tc-99 leave predictions worse than JEF2.2.
WPEC23 evaluations represent "state of the art" differential data. Their quality for individual nuclides is indicated in table 1 by the accuracy column where $\sigma$ is noted to be $4 \%$.

\section{Conclusion}

Fission product cross-sections for 12 nuclides from the JEF2.2 and JEFF3.1 libraries, plus new evaluations from WPEC23 have been assessed against results from the CERES integral benchmark. The thermal cross section and low energy resonance data for ${ }^{147,152} \mathrm{Sm}$ and ${ }^{155} \mathrm{Gd}$ are accurate to within $4 \%$. Similar data for ${ }^{109} \mathrm{Ag},{ }^{143} \mathrm{Nd}$ and ${ }^{149} \mathrm{Sm}$ are within $8 \%$ while ${ }^{95} \mathrm{Mo},{ }^{99} \mathrm{Tc},{ }^{103} \mathrm{Rh},{ }^{133} \mathrm{Cs}$ and ${ }^{145} \mathrm{Nd}$ are within three benchmark standard deviations at $\sim 12 \%$. The use of the 172 XMAS group scheme is adequate for all nuclides considered except ${ }^{153} \mathrm{Eu}$.

\section{References}

1. J.L. Rowlands (ed.), The JEF-2.2 Nuclear Data Library, JEFF Report 17 (OECD Publications, Paris, 2000).

2. N.T. Gulliford et al., Experimental Validation of Calculational Methods and Data for Burn-up Credit, in Conference program for Fifth International Conference on Nuclear Criticality Safety, ICNC'95 USA.

3. A Koning et al., The JEFF-3.1 Nuclear Data Library, JEFF Report 21 (OECD Publications, Paris, 2006).

4. P. Oblozinsky et al., Evaluated Data Library for the Bulk of the Fission Products, WPEC 23 (to be published by OECD Publications, Paris 2007) International Reactor Dosimetry File 2002 (IRDF-2002), IAEA Technical Reports Series No. 452, 2006.

5. R.E. MacFarlane, D.W. Muir, Los Alamos Report No. LA12740-M, 1994.

6. T.D. Newton et al., The next generation lattice code WIMS9, PHYSOR 2002, American Nuclear Society, USA. 